\title{
A SCREENING FOR NATURAL COLORANTS IN THE ZONGO VALLEY WITH PROBABLE ANTIOXIDANT AND/OR PHOTO-PROTECTOR ACTIVITIES
}

Sandra L. Ibáñez-Calero, Kelly E. Loayza Afonso, Ebbe L. Yapu Tapia, Jessica Lizarazu, Rodrigo Zeballos Espinoza and Teddy Solares Gironda

\begin{abstract}
Eleven plants were collected in the Zongo Valley following an organoleptic and chimio-taxonomic criteria of collection to find species with colorant and photo-protector properties. Brachyotum microdon, Monnina bridgesii and Souroubea fragilis present promising colorant attributes. In addition, B. microdon, Rumex acetosella and Fuchsia boliviana show important absorptions in the UV-B region while $S$. fragilis, Orthaea boliviensis, Senecio floccosus and Baccharis pentlandii have UV-A and UV-B absorptions. A series of phytochemical tests were performed to learn about the secondary metabolite profile in the collected species. This is the first work done and published for Souroubea fragilis, Orthaea boliviensis and Senecio floccosus.
\end{abstract}

Keywords: Zongo Valley, Photo-protector Properties, UV Absorption, Phytochemical Assays, Colorants, Brachyotum Microdon, Monnina Bridgesii, Orthaea Boliviensis, Senecio Floccosus and Souroubea Fragilis. 